\title{
Deterioration of exercise capacity after neonatal extracorporeal membrane
}

\section{oxygenation}

\author{
M.H.M. van der Cammen-van Zijp*,\#, , S.J. Gischler*,", W.C.J. Hop ${ }^{+}$, J.C. de \\ Jongste $^{\S}$, D. Tibboel*,\# and H. IJsselstijn*,\#
}

ABSTRACT: Extracorporeal membrane oxygenation (ECMO) provides life support in acute reversible cardiorespiratory failure. Assessment of long-term morbidity is essential to confirm survival advantage. This study aimed to assess exercise capacity in the first 12 yrs of life after neonatal ECMO, and to evaluate the effect of primary diagnosis, lung function or perinatal characteristics on exercise capacity.

120 children who, as neonates, underwent ECMO performed 191 reliable exercise tests according to the Bruce treadmill protocol at ages 5, 8 and/or 12 yrs between 2001 and 2010. Primary diagnoses were meconium aspiration syndrome $(n=66)$, congenital diaphragmatic hernia $(n=18)$ and other diagnoses $(n=36)$.

At ages 5, 8 and 12 yrs, ANOVA resulted in mean \pm SE standard deviation score endurance time on the treadmill of $-0.5 \pm 0.1,-1.1 \pm 0.1$, and $-1.5 \pm 0.2$, respectively, all significantly less than zero $(p<0.001)$. Exercise capacity declined significantly over time irrespective of the primary diagnosis.

Neonates treated with ECMO are at risk of decreased exercise capacity at school age. We therefore propose prolonged follow-up. Proactive advice on sports participation or referral to a physical therapist is recommended, especially when either the parents or the children themselves report impaired exercise capacity.

KEYWORDS: Chronic lung disease, congenital diaphragmatic hernia, exercise capacity, extracorporeal membrane oxygenation, follow-up

xtracorporeal membrane oxygenation (ECMO) is a pulmonary bypass technique providing life support in acute reversible cardiorespiratory failure when conventional management fails. It was originally suggested to avoid further lung injury from high oxygen concentration, volutrauma and barotrauma, and hence promote lung healing. In neonates, ECMO was first used over 30 yrs ago, mainly in congenital diaphragmatic hernia $(\mathrm{CDH})$, meconium aspiration syndrome (MAS), persistent pulmonary hypertension of the newborn or sepsis. A large trial in the UK concluded that there was a survival advantage of neonatal ECMO over conventional management without a concomitant increase in severe disability [1-3]. Thus, ECMO may be of benefit to infants with severe respiratory dysfunction who otherwise would have died. Detailed assessment of longerterm morbidity is considered essential to substantiate the reported survival advantage [4].

Neonates treated with ECMO may suffer longterm physical and developmental morbidity.
Severity of illness preceding ECMO, severe respiratory failure prior to ECMO and several factors during ECMO increase the risk of pulmonary dysfunction and cerebral damage [2, 5-8]. However, little is known about the effects of ECMO on exercise capacity. BOYKIN et al. [5] reported that the exercise tolerance of 10-15-yrold children treated with ECMO as neonates for MAS was similar to that of age-matched controls. However, HAMUTCU et al. [9] found that 48 children with a mean age of 11 yrs, treated with ECMO for various underlying diagnoses, had lower oxygen saturation with exercise and lower peak oxygen consumption than controls. The time course of these changes remains unclear, and to our knowledge, no longitudinal data of exercise tolerance after neonatal ECMO are available.

The aims of the present study were therefore to evaluate: whether children treated with ECMO at neonatal age have normal exercise capacity in the long term; how exercise capacity changes
AFFILIATIONS

*Depts of Intensive Care,

\#Paediatric Surgery,

-Rehabilitation Medicine and

Physical Therapy, and,

${ }^{\S}$ Paediatrics - Respiratory Medicine and Allergology, Erasmus MC-

Sophia Children's Hospital, and

+Dept of Biostatistics, Erasmus MC, Rotterdam, The Netherlands.

CORRESPONDENCE

M.H.M. van der Cammen-van Zijp Erasmus MC-Sophia Children's

Hospital

Intensive Care and Dept of Paediatric Surgery

and Dept of Rehabilitation Medicine and Physical Therapy

Room Sk 0327

Dr. Molewaterplein 60

3015 GJ Rotterdam

The Netherlands

E-mail: m.vandercammen@

erasmusmc.nl

Received:

May 172010

Accepted after revision:

April 082011

First published online:

May 032011

Online ISSN 1399-3003 
over time in these patients; and whether exercise capacity bears a relation to the primary diagnosis.

\section{METHODS}

\section{Patients}

A longitudinal follow-up study was conducted in neonates who received venoarterial (VA) ECMO support between January 1992 and July 2004 at the Intensive Care Unit of the Erasmus MCSophia Children's Hospital (Rotterdam, the Netherlands). The cohort was supplemented with five children who received VA ECMO in two other ECMO centres (Nijmegen, the Netherlands $(n=4)$ and Leuven, Belgium $(n=1))$. The latter both used the same inclusion criteria and treatment protocols as Erasmus MCSophia Children's Hospital. ECMO support was given in case of reversible severe respiratory failure and an estimated mortality risk of $>80 \%$ using the entry criteria reported by STOLAR et al. [10]. Entry criteria and exclusion criteria have previously been described by our group [11] and did not change during the study period. The study was part of a structured prospective postECMO follow-up programme initiated in 2001 that provides for regular assessments of lung function, growth and developmental parameters until age $18 \mathrm{yrs}[6,7]$. Based on the national consensus on neonatal follow-up and the Dutch Ministry of Health's requirement to provide relevant data, the assessment protocol is the standard of care in the Netherlands following ECMO. The Medical Ethical Review Board of Erasmus MC declared it had no objection to the implementation of this research project (approval reference MEC-2010-179). Parents of the participating children gave written permission for publication of the results of our follow-up.

\section{Procedures and study design}

The following clinical characteristics were recorded prospectively: underlying diagnosis, gestational age, birth weight, age at onset of ECMO, duration of ECMO support, mean airway pressure (MAP) and highest oxygenation index (OI) prior to ECMO, duration of mechanical ventilation before start of $\mathrm{ECMO}$, total duration of mechanical ventilation (including ECMO), duration of oxygen dependency and prevalence of chronic lung disease (CLD). According to the definition of JOBE and BANCALARI [12], CLD was defined as oxygen dependency at day 28. It was classified as mild, moderate or severe, based on the amount of oxygen needed at day 56 or at discharge, whichever was first [12].

The assessment protocol encompassed hospital visits at 5, 8 and 12 yrs of age. Medical assessment consisted of medical history taking, measurements of growth parameters and a standardised physical examination. Spirometry and an exercise test were performed when the child was clinically stable. Prior to the exercise test, parents estimated their child's fitness level as higher than, equal to or less than that of children the same age.

\section{Spirometry and exercise tests}

Spirometry was performed before and after bronchodilation with salbutamol. A dry rolling seal spirometer (Jaeger, Hoechberg, Germany) was used according to European Respiratory Society (ERS) criteria [13]. Three forced vital capacity (FVC) manoeuvres were performed and the best values of forced expiratory volume in $1 \mathrm{~s}$ (FEV1) and FVC were recorded. The children had been instructed to stop short-acting $\beta_{2}$-agonists $8 \mathrm{~h}$ before and long-acting $\beta_{2}$-agonists $24 \mathrm{~h}$ before assessment.

The children performed a graded maximum exercise test using a motor-driven treadmill (En Mill; Enraf Nonius, Rotterdam, the Netherlands) programmed for increases in angle of inclination and speed according to the Bruce protocol [14, 15]. The maximal endurance time (in minutes, precise to one decimal place) served as the criterion of exercise capacity. Before and during the test we monitored heart rate and transcutaneous oxygen saturation with a pulse oximeter (MARS (Motion Artifact Reduction System), type 2001; Respironics Novametrix, Murrysville, PA, USA). Heart rate of $\geqslant 185$ beats $\cdot \mathrm{min}^{-1}$ [16] or loss of coordination because of excessive fatigue was taken as maximal performance.

\section{Data analysis}

OI was calculated as ((MAP $\times$ inhaled oxygen fraction)/ arterial oxygen saturation) $\times 100$ [10]. The Dutch Growth Analyser, version 3.0 (Dutch Growth Foundation, Rotterdam, the Netherlands) was used to calculate standard deviation scores (SD-scores) for height, weight and body mass index (BMI), on the basis of Dutch reference values published in 2000 [17]. Adapted reference values were used for children of Moroccan or Turkish origin [18, 19]. SD-scores for spirometric data were calculated as the difference between observed and predicted value divided by the residual standard deviation from the reference values of STANOJEVIC et al. [20]. The SD-scores of the maximal endurance time were calculated using recent age-related reference values for healthy Dutch children $[14,15]$. SD-scores $<-1.96$ (2.5th percentile of the reference population) were considered abnormally low. Group comparisons were performed using the Mann-Whitney U-test. Longitudinal evaluation of the endurance times at 5, 8 and 12 yrs of age was performed using mixed-model ANOVA, which allows for missing data in an optimal way [21]. To investigate whether underlying diagnosis and other determinants had a significant influence on SD-score endurance time, we analysed diagnoses and the following components in the mixed model as covariates: gestational age, birth weight, OI, $\mathrm{MAP}$, time on ECMO, duration of ventilatory support before start of ECMO, total duration of ventilatory support, oxygen support after decannulation, the prevalence of CLD, SD-score for weight at follow up, SD-score for height at follow up, SDscore for BMI at follow up, SD-score for FEV1, SD-score for FEV1/FVC and sports participation. For continuous parameters with a log-normal distribution (OI, MAP, duration of ventilatory support before start of ECMO, total duration of ventilatory support), we transformed the data logarithmically in order to reduce the effect of outlying observations.

Values for two subgroups, $\mathrm{CDH}$ and MAS, were analysed separately. These two subgroups were considered of special interest. $\mathrm{CDH}$ is associated with abnormal lung development; infants with MAS form the largest subgroup of children with normal lung development. The other subgroups were small and more heterogeneous with respect to underlying disease. Statistical significance was accepted at a two-sided 5\% level for all tests. Statistical analyses were performed using SPSS 15.0 for Windows (SPSS Inc, Chicago, IL, USA). 


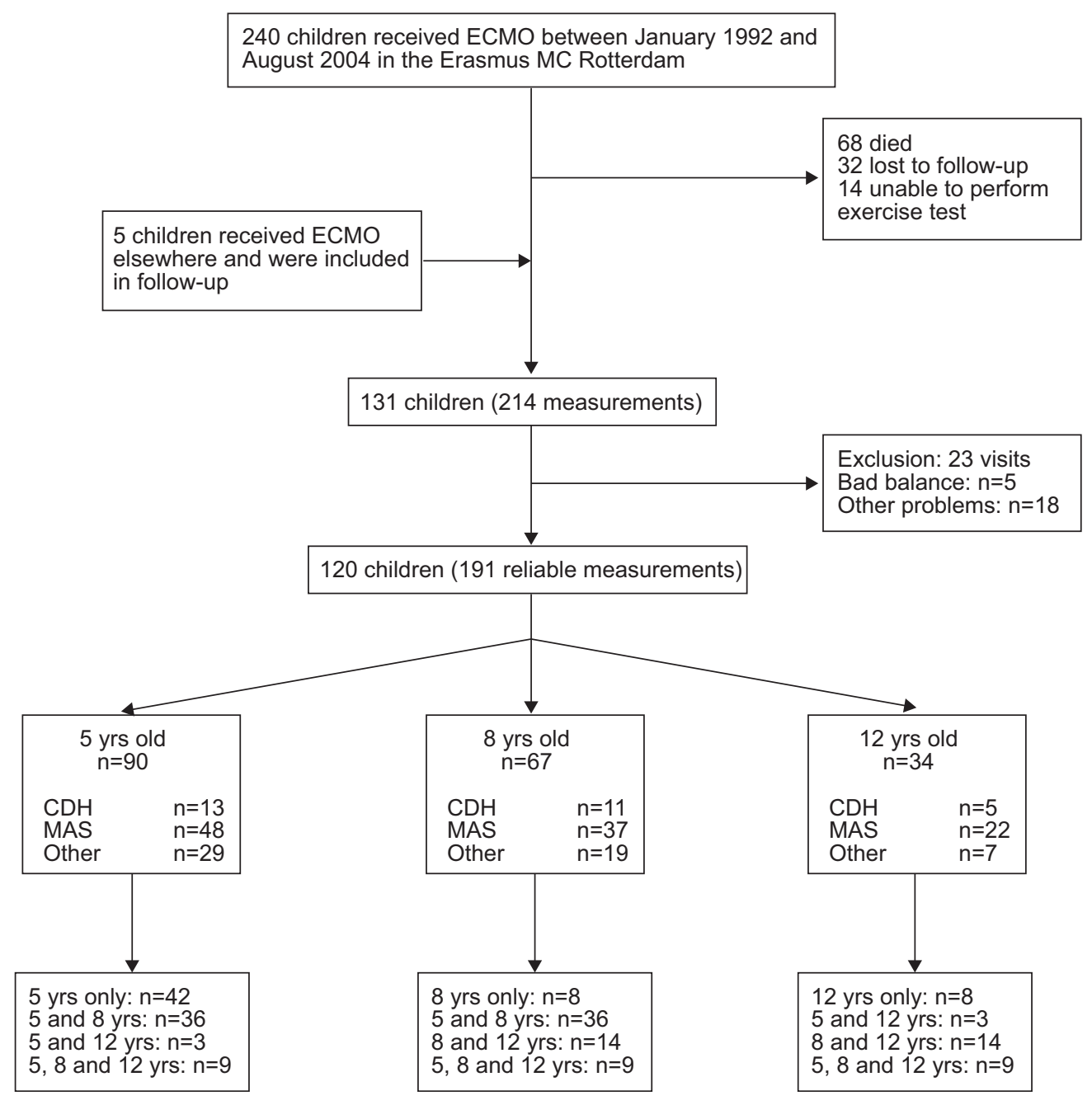

FIGURE 1. Flowchart of infants included in the follow-up programme. ECMO: extracorporeal membrane oxygenation; CDH: congenital diaphragmatic hernia; MAS meconium aspiration syndrome.

\section{RESULTS}

Between January 1992 and July 2004, 240 neonates received ECMO support within 28 days after birth at the Erasmus MCSophia Children's Hospital. 68 (28\%) of them died before age 5 yrs. $59 \%$ of those children were born with $\mathrm{CDH}$. In addition, five children who received ECMO support elsewhere were included in our follow-up programme. 32 children were lost to follow-up due to refusal to participate $(n=16)$, not being traceable $(n=10)$ or follow-up elsewhere $(n=10)$. Their baseline characteristics did not differ from those who were included in the final analysis.

145 children participated in our follow-up programme (82\% of all survivors). 14 children did not perform the exercise test because of neurological problems, such as hemiplegia and seizure disorder $(n=10)$; chromosomal disorder with mental retardation $(n=2)$; or behavioural problems $(n=2)$. These 14 children had been ventilated longer than those who were included in the analysis (Mann-Whitney U-test $p=0.027$ ).

Thus 131 children performed the Bruce treadmill test (214 measurements). We excluded 23 measurements because maximal performance could not be achieved due to balance problems $(n=5)$ and other reasons such as fear, pain in the legs and concentration problems $(n=18)$. The 11 children who did not perform any maximal exercise test had longer oxygen support and more severe CLD than the 120 participants (Mann-Whitney U-test $p=0.007$ and $p=0.012$, respectively). The final analysis included 191 exercise tests performed by 120 children $(68 \%$ of all survivors) (fig. 1). The primary diagnoses of these 120 children were MAS $(n=66), \mathrm{CDH}(n=18)$ and other diagnoses $(n=36)$. The diagnoses of the children in the "other diagnoses" group were: persistent pulmonary hypertension of the newborn $(n=20)$; sepsis $(n=7)$; pneumonia $(n=6)$; cardiorespiratory problems $(n=2)$; and congenital cystic adenomatoid malformation of the lung $(n=1)$. The perinatal characteristics and ECMO treatment characteristics of all survivors are presented in table 1. Inhaler medication was used by $3.4 \%$ of the 5 -yr-olds, $10.4 \%$ of the 8 -yr-olds and $5.9 \%$ of the 12 -yr-olds.

At time of follow-up, the mean SD-score for FEV1 was significantly below zero at ages 5,8 and $12 \mathrm{yrs}$. This also applies to mean SD-score for FEV1/FVC at ages 8 and 12 yrs. The mean change in FEV1 from baseline was $8.6 \%, 6.3 \%$, and $7.2 \%$ at 5,8 , and $12 \mathrm{yrs}$, respectively. We observed significant 
TABLE 1 Perinatal and extracorporeal membrane oxygenation (ECMO) characteristics

All survivors

Participants
Lost to follow-up

Inability to perform No maximal performance exercise test

\begin{tabular}{|c|c|c|c|c|c|}
\hline Subjects $n$ & 177 & 120 & 32 & 14 & 11 \\
\hline Males/females $\mathbf{n}$ & $94 / 83$ & $60 / 60$ & $21 / 11$ & $7 / 7$ & $7 / 4$ \\
\hline \multicolumn{6}{|l|}{ Primary diagnosis } \\
\hline $\mathrm{CDH}$ & $29(17)$ & $18(15)$ & $3(9)$ & $4(29)$ & $4(36)$ \\
\hline Other diagnoses & $52(29)$ & $36(30)$ & $12(38)$ & $4(29)$ & $1(9)$ \\
\hline Gestational age weeks & $39.7 \pm 1.9$ & $39.8 \pm 1.8$ & $39.7 \pm 1.9$ & $38.7 \pm 2.3$ & $40.5 \pm 1.5$ \\
\hline MAP $\mathrm{cmH}_{2} \mathrm{O}$ & $20.0 \pm 4.5$ & $19.9 \pm 4.4$ & $20.9 \pm 3.6$ & $21.7 \pm 5.1$ & $21.7 \pm 6.9$ \\
\hline Pre-ECMO treatment with NO & $115(65)$ & $74(62)$ & $22(69)$ & $10(71)$ & $9(82)$ \\
\hline Age before start of ECMO $\mathrm{h}$ & $24(4-600)$ & $27(5-398)$ & $25(5-288)$ & $20(4-600)$ & $24(5-41)$ \\
\hline Time on ECMO $\mathrm{h}$ & $132(24-510)$ & $131(24-369)$ & $133(48-347)$ & $172(61-510)$ & $140(58-220)$ \\
\hline Time on ventilatory support days & $12(1-130)$ & $11(1-70)^{\#}$ & $13(5-40)$ & $20(8-130)^{\#}$ & $9(6-50)$ \\
\hline \multicolumn{6}{|l|}{ Time on oxygen support } \\
\hline Unknown & $25(14)$ & $12(10)$ & $9(28)$ & $3(21)$ & $1(9)$ \\
\hline \multicolumn{6}{|l|}{ CLD } \\
\hline None & $125(71)$ & $95(79)^{+}$ & $18(56)$ & $7(50)$ & $5(46)^{+}$ \\
\hline Mild & $17(10)$ & $7(6)$ & $6(19)$ & $1(7)$ & $3(27)$ \\
\hline Moderate & $4(2)$ & $2(2)$ & $1(3)$ & $1(7)$ & $0(0)$ \\
\hline Severe & $14(8)$ & $9(7)$ & $1(3)$ & $2(14)$ & $2(18)$ \\
\hline Unknown & $17(9)$ & 7 (6) & $6(19)$ & 3 (22) & $1(9)$ \\
\hline
\end{tabular}

Data are presented as $n(\%)$, mean \pm SD or median (range), unless otherwise stated. MAS: meconium aspiration syndrome; CDH: congenital diaphragmatic hernia; MAP mean airway pressure; NO: nitric oxide; CLD: chronic lung disease. ${ }^{*}: p=0.027$, Mann-Whitney U-test, difference in ventilatory support between children who were unable to perform exercise test and participants; ${ }^{\top}: p=0.007$, Mann-Whitney U-test, difference in oxygen support between children without maximal performance and participants; ${ }^{+}: p=0.012$, Mann-Whitney U-test, difference in CLD between children without maximal performance and participants.

reversibility of airflow obstruction (i.e. a change in FEV1 $>11 \%$ [22]) in 13, 12 and six children aged 5, 8 and 12 yrs, respectively. The characteristics at time of follow-up of the children of the study group are presented in table 2 .

\section{Exercise capacity}

At ages 5, 8 and 12 yrs, ANOVA resulted in mean \pm SE SD-score endurance time on the Bruce treadmill protocol of $-0.5 \pm 0.1$, $-1.1 \pm 0.1$ and $-1.5 \pm 0.2$, respectively, all significantly less than 0 (all $\mathrm{p}<0.001$ ). Standard deviations of the mean SD-score endurance time at the three measurement points were 1.06, 1.18 and 1.18 , respectively. The mean difference between SDscore endurance time at ages 8 and 5 yrs was $-0.5(95 \% \mathrm{CI}$ $-0.9-0.2)$; that between ages 12 and 8 yrs was $-0.4(95 \% \mathrm{CI}$ $-0.8-0.0)$. The SD-score was abnormally low $(<-1.96)$ in six $(7 \%)$ out of 90 measurements at age 5 yrs, in $10(15 \%)$ out of 67 measurements at age $8 \mathrm{yrs}$ and in 12 (35\%) out of 34 measurements at age 12 yrs. At the end of the test, heart rate was reliably recorded in $156(82 \%)$ measurements and the 10th and 90th percentiles were 168 and 200 beats $\cdot \mathrm{min}^{-1}$, respectively. Technical problems precluded reliable recording of heart rate in the final stage of the test for the other 35 measurements, but, based on heart rate in the pre-final stage or loss of coordination, we considered performance in those measurements to be maximal. In five out of 156 measurements, there was a decrease in transcutaneous oxygen saturation of $\geqslant 5 \%$ from baseline (two out of 29 measurements in children with $\mathrm{CDH}$ and three out of 107 measurements in children with MAS). None of the children had transcutaneous oxygen saturation $<90 \%$.

Figure 2 shows the longitudinal evaluation of the SD-score for endurance time at ages of 5, 8 and 12 yrs for the total group and for the different initial diagnoses. 62 children underwent at least two measurements at different ages. All together, there were 80 paired measurements. 34 of these paired measurements showed a deterioration of $>1$ in SD-score. In 41 paired measurements, the change in SD-score ranged between -1 and +1 (suggesting no significant change in exercise capacity). Improvement in SD-score of $\geqslant 1$ was observed five times. The mean outcome at age 5 yrs was higher than at ages 8 and 12 yrs (both $p \leqslant 0.001$ ), with a marginal difference between ages 8 and 12 yrs $(p=0.050)$. The underlying diagnosis had no significant influence. Further analysis using ANOVA did not show significant relationships with: time on ECMO; duration of ventilatory support before start of ECMO; total duration of ventilatory support; oxygen support after decannulation; 


\begin{tabular}{|c|c|c|c|}
\hline Subjects $n$ & 90 & 67 & 34 \\
\hline Males/females $n$ & $43 / 47$ & $36 / 31$ & $16 / 18$ \\
\hline $\mathrm{CDH}$ & $13(15)$ & $11(16)$ & $5(15)$ \\
\hline MAS & 48 (53) & 37 (55) & 22 (65) \\
\hline Other & $29(32)$ & $19(29)$ & $7(20)$ \\
\hline SD-score for height & $-0.22 \pm 1.28$ & $-0.15 \pm 1.08$ & $0.04 \pm 1.13$ \\
\hline SD-score for weight & $-0.28 \pm 1.55$ & $0.00 \pm 1.37$ & $0.05 \pm 1.15$ \\
\hline SD-score for BMI & $-0.19 \pm 1.26$ & $0.14 \pm 1.24$ & $0.10 \pm 1.07$ \\
\hline \multicolumn{4}{|c|}{ Parental estimation of fitness level } \\
\hline Better than peers & $5(6)$ & $4(6)$ & $2(6)$ \\
\hline Similar to peers & $63(70)$ & $47(70)$ & $22(65)$ \\
\hline Worse than peers & $19(21)$ & $16(24)$ & $10(29)$ \\
\hline Missing & $3(3)$ & $0(0)$ & $0(0)$ \\
\hline \multicolumn{4}{|c|}{ Physical therapy at home } \\
\hline Yes & $6(7)$ & $3(5)$ & $0(0)$ \\
\hline No & $84(93)$ & $64(95)$ & $34(100)$ \\
\hline SD-Score for FEV 1 & $-0.54 \pm 1.11^{\#, \star \star \star}$ & $-0.64 \pm 1.29^{\circ * \star \star *}$ & $-1.11 \pm 1.54^{+, \star \star \star}$ \\
\hline SD-score for $\mathrm{FEV}_{1} / \mathrm{FVC}$ & $-0.11 \pm 1.44^{\#}$ & $-0.70 \pm 1.15^{\circ, \star \star \star}$ & $-1.03 \pm 1.22^{+, * * *}$ \\
\hline
\end{tabular}

Data are presented as $n(\%)$ or mean \pm SD, unless otherwise stated. $\mathrm{CDH}$ : congenital diaphragmatic hernia; MAS: meconium aspiration syndrome; SD-Score: standard deviation score; BMI: body mass index; FEV1: forced expiratory volume in $1 \mathrm{~s}$; FVC: forced vital capacity. ${ }^{\#}: n=62 ;{ }^{\circ}: n=53 ;{ }^{+}: n=28 ;{ }^{* \star \star}$ : $p \leqslant 0.001$, one-sample t-test, mean value significantly different from zero.

prevalence of CLD; SD-score for weight at follow-up; SD-score for height at follow-up; SD-score for BMI at follow-up; SD-score for FEV1; SD-score for FEV1/FVC; and sports participation (data not shown). The levels of exercise capacity as estimated by the parents were positively correlated with the measured endurance SD-scores $(\mathrm{p}=0.002)$.

\section{DISCUSSION}

We evaluated exercise capacity at ages 5, 8 and 12 yrs in children who had undergone neonatal ECMO treatment. Exercise capacity declined significantly over time, irrespective of the underlying primary diagnosis. Parents' estimations of the children's exercise capacity correlated positively with the endurance SD-scores. None of the clinical characteristics correlated with exercise tolerance.

14 children did not perform the exercise test because of neurological or behavioural problems. These 14 children seemed to be the sickest individuals, as reflected by the longer duration of ventilation, and should therefore be considered as poor outcome. BOYKIN et al. [5] tested the exercise capacity of 10-15-yrold children who as neonates had received ECMO treatment for MAS [5]. The cross-sectional study revealed that the 17 ECMOtreated children had similar aerobic capacity as age-matched healthy controls. Duration of oxygen use following decannulation proved the most significant factor in predicting long-term pulmonary outcome. In our study, the mixed linear model analysis revealed deterioration of exercise capacity irrespective of the underlying diagnosis. Our study population presumably included more children with initial pulmonary hypoplasia and residual pulmonary sequelae, because BOYKIN et al. [5] studied only MAS patients, who have intrinsically normal lungs.

HAMUTCU et al. [9] showed that 48 children (mean age 11.1 yrs), treated with ECMO for various conditions, including MAS and $\mathrm{CDH}$, had lower peak oxygen consumption than healthy agematched controls. Differences in the protocols used hamper comparison of the results. Nevertheless, we would like to point out that the oxygen desaturation (arterial oxygen saturation measured by pulse oximetry $<90 \%$ ) observed during exercise in almost $25 \%$ of the ECMO-treated children in the study of HAMUTCU et al. [9] was not observed in any of our participants. Unlike HAMUTCU et al. [9], we did not measure gas exchange parameters for various reasons. First, wearing a mask may lead to loss of cooperation and to submaximal results, especially in younger children. Secondly, CumMing et al. [23] reported a strong correlation between maximal endurance time and maximal oxygen uptake. They concluded that maximal endurance time might be used as a sole criterion of exercise capacity.

Recently, Gischler et al. [24], from our group, evaluated maximal exercise performance in 16 5-yr-old children born 


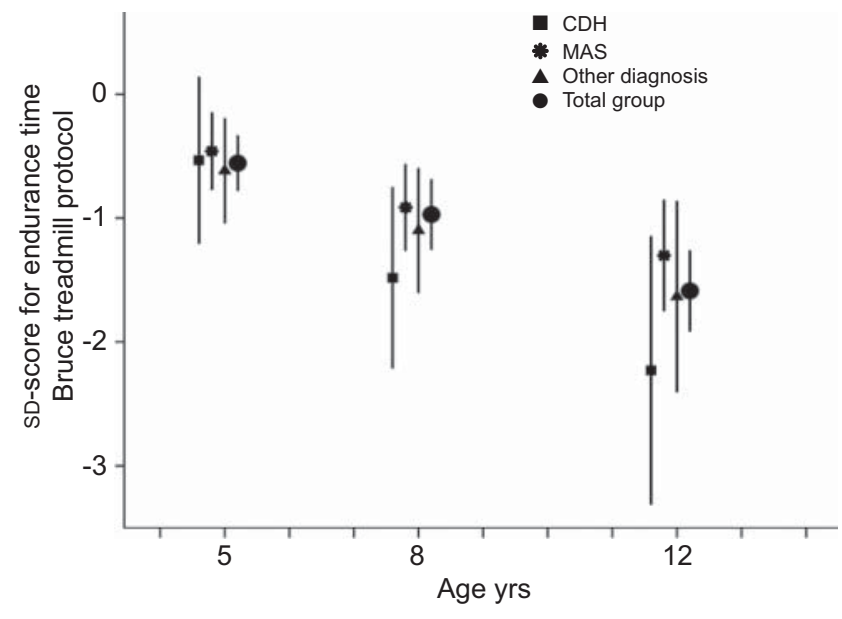

FIGURE 2. Exercise capacity at ages 5, 8 and $12 \mathrm{yrs}$. Data are presented as ANOVA estimates of mean values, with 95\% confidence intervals. SD-Score: standard deviation score; $\mathrm{CDH}$ : congenital diaphragmatic hernia; MAS: meconium aspiration syndrome.

with $\mathrm{CDH}$, of whom nine had undergone neonatal ECMO treatment. The mean SD-score for endurance time was -0.84 (also significantly different from zero, $\mathrm{p}=0.012$ ) [24]. In a study by PEETSOld et al. [25], 36 out of 53 survivors of CDH who had not received ECMO treatment (mean \pm SD age $11.9 \pm 3.5 \mathrm{yrs}$ ) reliably performed the Bruce treadmill test. In most of them, the exercise capacity agreed with the reference values of BINKHORST et al. [26] established in 1987. There was a positive but small correlation between the SD-score for peak oxygen uptake and the SD-score for FEV1 $\left(r^{2}=0.27 ; p=0.001\right)$. In our study, the correlation between maximal endurance time and SD-score for FEV1 was not significant. ECMO offers survival to those children with $\mathrm{CDH}$ with more severe lung hypoplasia. The children in the study of PEeTsOld et al. [25] who did not need ECMO at all represent survivors with a milder form of $\mathrm{CDH}$.

VRIJLANDT et al. [27] reported on exercise capacity in young adults who had been born prematurely and in age-matched controls. As bronchopulmonary dysplasia was over-represented in the born-prematurely group, the exercise capacity of participants with and without bronchopulmonary dysplasia was compared. No significant difference was found.

There is a question over whether bronchodilators would have improved our results. We do not think so, because we observed a significant reversibility of airway obstruction in only a small number of children $(n=31)$. In $20(64.5 \%)$ of them, lung function assessment with bronchodilation was performed prior to the exercise test. So, better performance during the exercise test could have been expected in 11 children only.

Previously, we have found balance-skill problems in 5-yr-old children treated with neonatal ECMO [7]. These could perhaps (partly) explain the decline in exercise capacity between ages 5 and 8 yrs. In the present study, 5-yr-old children were allowed to hold the guardrail to maintain body position near the centre of the moving belt, unlike older children [14, 15]. Hence, part of the deterioration between ages 5 and 8 yrs could be explained by possible balance problems. However, this cannot explain deterioration between ages 8 and 12 yrs.

Another explanatory factor could be lack of exercise; however, reported sports participation had increased at $8 \mathrm{yrs}$; this seems to contradict lack of exercise as explanatory factor. At $12 \mathrm{yrs}$, on the contrary, $26 \%$ of the 12 -yr-old children did not report sports participation.

Regrettably, we have no data from computed tomography scans, so progressive fibrosis as a reason for deterioration over time cannot be ruled out.

In our study group, 18 patients had $\mathrm{CDH}$ with pulmonary hypertension and 20 were treated with ECMO for persistent pulmonary hypertension. In both conditions, pulmonary vasoreactivity may persist and result in ventilation-perfusion mismatch. We did not perform cardiac evaluations to confirm this speculation. For CDH patients, ventilation-perfusion mismatch has been described previously [28].

In contrast to the UK Collaborative ECMO trial [1], our study was not set up as a randomised study with controls suffering from severe neonatal cardiorespiratory failure who were treated conventionally. With only two ECMO centres in the Netherlands, covering a relatively small geographical area, the large majority of neonates with similar severity of illness who are not born prematurely (i.e. born after 34 weeks' gestation with birth-weight $>2,000 \mathrm{~g}$ ) are treated with ECMO. Therefore, it was not possible to include a sufficient number of children who survived without ECMO to serve as a control group, since use of historical data is not desirable. Recruiting controls from other countries with less easy access to ECMO treatment could have been an option. However, differences in the definitions of optimal care for specific diseases and differences in quality of care will almost certainly create bias.

At follow-up at age 7 yrs, the conventionally treated children in the UK Collaborative ECMO trial had more respiratory morbidity than those treated with ECMO [3]. Thus, we speculate that imaginary disease-matched controls in our study would have performed worse than the ECMO-treated children.

Another possible limitation of our study is the lack of healthy controls. We do not think that this invalidates our findings, as our study aimed to prospectively evaluate exercise capacity with advancing age. We used reference data for the Bruce treadmill protocol recently obtained in healthy Dutch children $[14,15]$. Furthermore, testing at the different ages was done with exactly the same protocol and equipment as in that study and for the most part even by the same investigator.

In conclusion, neonates treated with ECMO are at risk of decreased exercise capacity at school age. We therefore propose prolonged follow-up. Proactive advice on sports participation or referral to a physical therapist is recommended, especially when either the parents or the children themselves report impaired exercise capacity.

\section{SUPPORT STATEMENT}

The present study was supported by Fonds Nuts-Ohra and Stichting Swart-van Essen Fonds, Rotterdam, the Netherlands. 


\section{STATEMENT OF INTEREST}

None declared.

\section{ACKNOWLEDGEMENTS}

K. Hagoort (Dept of Paediatric Surgery, Erasmus MC-Sophia Children's Hospital, Rotterdam, the Netherlands) provided editorial advice.

\section{REFERENCES}

1 UK Collaborative ECMO Trail Group. UK collaborative randomised trial of neonatal extracorporeal membrane oxygenation. Lancet 1996; 348: 75-82.

2 Bennett CC, Johnson A, Field DJ, et al. UK collaborative randomised trial of neonatal extracorporeal membrane oxygenation: follow-up to age 4 years. Lancet 2001; 357: 1094-1096.

3 McNally H, Bennett CC, Elbourne D, et al. United Kingdom collaborative randomized trial of neonatal extracorporeal membrane oxygenation: follow-up to age 7 years. Paediatrics 2006; 117: e845-e854.

4 Beardsmore CS, Westaway J, Killer $\mathrm{H}$, et al. How does the changing profile of infants who are referred for extracorporeal membrane oxygenation affect their overall respiratory outcome? Paediatrics 2007; 120: e762-e768.

5 Boykin AR, Quivers ES, Wagenhoffer KL, et al. Cardiopulmonary outcome of neonatal extracorporeal membrane oxygenation at ages 10-15 years. Crit Care Med 2003; 31: 2380-2384.

6 Hanekamp MN, Mazer P, van der Cammen-van Zijp MH, et al. Follow-up of newborns treated with extracorporeal membrane oxygenation: a nationwide evaluation at 5 years of age. Crit Care 2006; 10: R127.

7 Nijhuis-van der Sanden MW, van der Cammen-van Zijp MH, Janssen AJ, et al. Motor performance in five-year-old extracorporeal membrane oxygenation survivors: a population-based study. Crit Care 2009; 13: R47.

8 Glass P, Bulas DI, Wagner AE, et al. Severity of brain injury following neonatal extracorporeal membrane oxygenation and outcome at age 5 years. Dev Med Child Neurol 1997; 39: 441-448.

9 Hamutcu R, Nield TA, Garg M, et al. Long-term pulmonary sequelae in children who were treated with extracorporeal membrane oxygenation for neonatal respiratory failure. Paediatrics 2004; 114: 1292-1296.

10 Stolar CJ, Snedecor SM, Bartlett RH. Extracorporeal membrane oxygenation and neonatal respiratory failure: experience from the extracorporeal life support organization. J Pediatr Surg 1991; 26: 563-571.

11 Hofhuis W, Hanekamp MN, IJsselstijn $\mathrm{H}$, et al. Prospective longitudinal evaluation of lung function during the first year of life following extracorporeal membrane oxygenation. Pediatr Crit Care Med 2011; 12: 159-164.

12 Jobe AH, Bancalari E. Bronchopulmonary dysplasia. Am J Respir Crit Care Med 2001; 163: 1723-1729.
13 Miller MR, Hankinson J, Brusasco V, et al. Standardisation of spirometry. Eur Respir J 2005; 26: 319-338.

14 van der Cammen-van Zijp MH, IJsselstijn $\mathrm{H}$, Takken $\mathrm{T}$, et al. Exercise testing of pre-school children using the Bruce treadmill protocol: new reference values. Eur J Appl Physiol 2010; 108: 393-399.

15 van der Cammen-van Zijp MH, van den Berg-Emons RJ, Willemsen SP, et al. Exercise capacity in Dutch children: new reference values for the Bruce treadmill protocol. Scand J Med Sci Sports 2010; 20: e130-e136.

16 Karila C, de Blic J, Waernessyckle S, et al. Cardiopulmonary exercise testing in children: an individualized protocol for workload increase. Chest 2001; 120: 81-87.

17 Fredriks AM, van Buuren S, Burgmeijer RJ, et al. Continuing positive secular growth change in The Netherlands 1955-1997. Pediatr Res 2000; 47: 316-323.

18 Fredriks AM, van Buuren S, Jeurissen SE, et al. Height, weight, body mass index and pubertal development reference values for children of Turkish origin in the Netherlands. Eur J Pediatr 2003; 162: 788-793.

19 Fredriks AM, van Buuren S, Jeurissen SE, et al. Height, weight, body mass index and pubertal development references for children of Moroccan origin in The Netherlands. Acta Paediatr 2004; 93: 817-824.

20 Stanojevic S, Wade A, Stocks J, et al. Reference ranges for spirometry across all ages: a new approach. Am J Respir Crit Care Med 2008; 177: 253-260.

21 Fitzmaurice G, Laird N, Ware J. Applied Longitudinal Analysis. 1st Edn. Hoboken, John Wiley \& Sons, 2004.

22 Casan P, Roca J, Sanchis J. Spirometric response to a brochodilator. Reference values for healthy children and adolescents. Bull Eur Physiopathol Respir 1983; 19: 567-569.

23 Cumming GR, Everatt D, Hastman L. Bruce treadmill test in children: normal values in a clinic population. Am J Cardiol 1978; 41: 69-75.

24 Gischler SJ, van der Cammen-van Zijp MH, Mazer P, et al. A prospective comparative evaluation of persistent respiratory morbidity in esophageal atresia and congenital diaphragmatic hernia survivors. J Pediatr Surg 2009; 44: 1683-1690.

25 Peetsold MG, Heij HA, Nagelkerke AF, et al. Pulmonary function and exercise capacity in survivors of congenital diaphragmatic hernia. Eur Respir J 2009; 34: 1140-1147.

26 Binkhorst RA, van't Hof MA, Saris WHM. Maximale inspanning door kinderen; referentiewaarden voor 6-18 jarige meisjes en jongens. [Maximal exercise in children; reference values girls and boys, 6-18 years of age.] The Hague, Nederlandse Hartstichting, 1992.

27 Vrijlandt EJ, Gerritsen J, Boezen HM, et al. Lung function and exercise capacity in young adults born prematurely. Am J Respir Crit Care Med 2006; 173: 890-896.

28 Hayward MJ, Kharasch V, Sheils C, et al. Predicting inadequate long-term lung development in children with congenital diaphragmatic hernia: an analysis of longitudinal changes in ventilation and perfusion. J Pediatr Surg 2007; 42: 112-116. 